Peguera Carré, M.C., Curto Reverte, A., lanos, M.A. y Coiduras Rodríguez, J.L.(2021). Evaluación de narrativas en formación dual docente: diseño y experimentación del instrumento SCAN. Revista de Investigación Educativa, 39(1), 111-130.

DOI: http://dx.doi.org/10.6018/rie.415271

\title{
Evaluación de narrativas en formación dual docente: diseño y experimentación del instrumento SCAN'
}

\section{Evaluating written works in dual teacher education: SCAN instrument design and experimentation}

\author{
Maria Carme Peguera Carré*,Andreu Curto Reverte*, Maria Adelina lanos** y Jordi Lluís \\ Coiduras Rodríguez* \\ *Departament de pedagogia. \\ **Departament de psicologia. \\ Facultat d'Educació, Psicologia i Treball Social. Universitat de Lleida (España).
}

\section{Resumen}

En el artículo se presenta la construcción y validación del instrumento de evaluación Sistema de Categorías para el Análisis de Narrativas (SCAN) integrado en un dispositivo de formación dual. SCAN facilita una evaluación convergente por los distintos agentes de la Formación Dual Universitaria (FDU) en un grado de Educación Primaria: profesorado universitario, Docentes en Formación Inicial (DFI) y mentores escolares.

Este estudio de caso analiza con dos puntos de control la evolución de las narrativas sobre la práctica educativa en la escuela. Se presta especial atención a la relación teoría-práctica mediante la evaluación de cuatro dimensiones: Descripción, Comprensión, Proyección y Aspectos formales.

El análisis de las narraciones de 150 DFI muestra una mejora significativa entre el pretest y el postest, especialmente relevante en la dimensión Proyección, evidenciándose en las categorías: Competencias Docentes, Intervención Docente y Oportunidades de Desarrollo.

Correspondencia: Maria Carme Peguera Carré, mariacarme.peguera@udl.cat, Av. de l'Estudi General, 4, 25001, Lleida.

1 Este trabajo ha recibido la ayuda de la Agencia de Ayudas para la Investigación y Universidades (AGAUR) dependiente del Departamento de Economía y de Sociedad del Conocimiento: 2015 ARMIF 00022, 2017 ARMIF 00028. Así como la ayuda de la Universidad de Lleida en el Programa de Promoción de la Investigación (2019) y del Ministerio Ciencia, Innovación y Universidades (BEAGAL18/00234). 
Se concluye que el procedimiento formativo descrito contribuye al desarrollo de la Visión Profesional desde una revisión crítica de la práctica. SCAN muestra en este estudio su eficiencia en el análisis y la evaluación de las narrativas de los DFI.

Palabras clave: programa de alternancia estudios-trabajo; formación inicial de maestros; relación teoría-práctica; rúbrica de evaluación.

\begin{abstract}
The article presents the construction and validation of the evaluation instrument called Narratives Analysis Categories System (SCAN) integrated in a dual initial training of teachers. SCAN facilitates a convergent evaluation by the different actors of the Dual University Training (FDU) in the degree of Primary Education: the university teaching staff, the Teachers in Initial Training (DFI) and the school mentors.

This case study analyses with two checkpoints the evolution of narratives about educational practices in schools. It focuses mainly on the relationship between theory and practice through the assessment of four dimensions: Description, Comprehension, Projection and Formal Aspects.

The narrative analysis of 150 DFIs shows a significant improvement between the pre-test and post-test, especially relevant in the Projection dimension. This is evidenced in the following categories: Teaching Competencies, Teaching Intervention and Development Opportunities.

In conclusion, the training with narratives contributes to the development of Professional Vision from a critical review of practice. In this study SCAN shows its efficiency in the analysis and evaluation of the DFIs narratives.

Keywords: cooperative education; preservice teacher education; theory practice relationship; scoring rubrics.
\end{abstract}

\title{
Introducción
}

La práctica es un componente fundamental en los planes de estudio de los Grados de Educación. El centro escolar se entiende como un espacio profesionalizador donde los Docentes en Formación Inicial (DFI) deben movilizar los recursos internos y externos para responder adecuadamente a situaciones educativas reales (Le Boterf, 2002). La inmersión y experiencia en la escuela contribuyen al desarrollo de la dimensión colectiva como parte de una comunidad profesional (Goyette \& Martineau, 2018).

En la Formación Dual Universitaria (FDU) el acompañamiento, el feedback constante y la supervisión facilitan el ejercicio progresivo de las competencias profesionales en las prácticas (Álvarez-Santullano \& De Prada, 2018). Además, Bucheton y Soulé (2009) y Danielson (2013) argumentan la necesidad de articular estrategias para que los DFI se inicien en la deconstrucción de la complejidad de la labor docente desde una perspectiva multidimensional. Autoras como Leroux y Portelance (2018) afirman que la integración teoría-práctica ayuda a comprender la experiencia formativa y a reconocer las situaciones relevantes. La atención crítica en el aula (metodología, uso de recursos, organización, clima y resultados de aprendizaje) permite tomar conciencia sobre los aspectos esenciales y ensayar alternativas en los métodos de actuación (Korthagen, 2017). 


\section{Universidad y escuela, escenarios para la integración de aprendizajes}

En las últimas décadas una de las preocupaciones esenciales de la investigación se ha situado en la vinculación de la teoría-práctica y en el diseño de programas adecuados para esta conexión (Korthagen, 2017; Ferrández-Berrueco \& Sánchez-Zarazaga, 2019; Sarceda-Gorgoso \& Rodicio-García, 2017). Los planes de estudio derivados del Espacio Europeo de Educación Superior han permitido ampliar el Prácticum y plantear estrategias de análisis mediante metodologías de observación y de revisión de la experiencia. El reto sigue entendiéndose como complejo por la ambigüedad en la interpretación del problema y las distintas posibilidades de relacionar teorías y prácticas en ambos sentidos.

Los distintos modelos formativos se ven interpelados por esta cuestión, desde la formación basada en la escuela o "school-based teacher education", la formación basada en la investigación o "research-based teacher education" (Munthe \& Rogne, 2015) y la formación realística o "realistic teacher education" (Melief, Tigchelaar, Korthagen \& van Rijswijk, 2010). En este tercer modelo se considera esencial atender al enfoque clínico de la práctica agregando a la formación pedagogías de reflexión e investigación. Para ello es necesario vencer las tradicionales divisiones históricas que subyacen entre universidad y escuela, desarrollar una cultura de la reflexión y de conexión entre experiencia y marcos teóricos. Desde la formación realística se enfatiza la interactividad del aprendizaje en la universidad con el derivado del contexto escolar.



Figura 1. Distribución temporal de los contextos formativos del GEP-dual.

El Grado de Educación Primaria dual (GEP-dual), donde se implementa nuestro estudio, es una interpretación del modelo de formación realística. En la modalidad dual se intensifica la actividad práctica, con una distribución de la presencialidad de un $40 \%$ en los centros escolares y de un $60 \%$ en la Universidad. A lo largo de los distintos cursos el alumnado dual debe asistir a distintos tipos de escuelas, urbanas, rurales y de máxima complejidad, como una estrategia para conocer la diversidad de los públicos escolares y aprehender el ejercicio docente en ellas (Figura 1).

Las cuestiones que emergen de la práctica constituyen una fuente fundamental para el análisis y la movilización del saber teórico. El carácter integrativo del modelo 
requiere un elevado nivel de exigencia y compromiso para todas las partes implicadas, con un enfoque nuevo en el diseño del plan de estudios (Correa-Molina, 2015). Coiduras, Correa-Molina, Boudjaoui y Curto (2017) consideran que la FDU precisa ayudas orientadas a la integración teórico-práctica desde lógicas deductivas e inductivas para promover la reflexividad.

\section{Las narrativas en el desarrollo de la Visión Profesional}

Los diarios reflexivos, de campo y los portafolios, con seguimiento y supervisión, han sido las estrategias más utilizadas para estimular la reflexión sobre la propia actuación profesional en la formación inicial (Carandell, Keim \& Tigchelaar, 2010). Uno de los focos de interés se ha situado en la observación de la apropiación del conocimiento científico de la comunidad docente, como dimensión social de la educación (Podolskiy, 2019). Alguna autora como Beauchamp (2015) identifica la reflexividad como la metacompetencia necesaria del DFI para supervisar su aprendizaje durante la formación y desarrollar la identidad profesional docente (Hanna, Oostdam, Severiens \& Zijlstra, 2020). Vinculamos la reflexividad con el análisis y el aprender a ver, como una forma de acercarse a la realidad desde el conocimiento empírico y el conocimiento formalizado, necesarios para una toma de decisiones más informada antes, durante o después de la actuación docente.

Investigadoras de metodología observacional como Seidel y Stürmer (2014) exponen que estrategias de análisis de la práctica educativa favorecen el desarrollo de la Visión Profesional. Desde esta perspectiva, impulsar la observación, la narración y la interpretación de situaciones reales beneficia la reflexividad. Las narrativas trasladan aquello que se ha observado desde la atención selectiva a la interpretación y argumentación (van Es, Cashen, Barnhart \& Auger, 2017). Reconocemos un interés común y fundamental en la literatura en la adquisición de una visión crítica, orientada al desarrollo docente.

La capacidad de argumentación sobre la propia práctica es fundamental en el desarrollo de la Visión Profesional. Algunos referentes han defendido la necesidad de instrumentalizar la evaluación de las narrativas para objetivar la calidad y conocer los niveles de reflexión (Carandell et al., 2010). A la vez, reconocen en estos instrumentos las posibilidades de ser utilizados para la autoevaluación y autorregulación del rol profesional.

\section{Instrumentos para el análisis de las narrativas}

En los últimos años, la evaluación de la reflexividad a partir de textos ha motivado la producción de distintas rúbricas como la de Alsina et al. (2017) y Domingo y Gómez (2014).

Ward y McCotter (2004) idearon una rúbrica para que los estudiantes pudieran evaluar sus textos bajo tres dimensiones: el foco o preocupación, la argumentación o investigación y el planteamiento de cambio de la práctica con una nueva visión. Watts y Lawson (2009) observaron que la rúbrica presentada por Ward y McCotter (2004) permite que los estudiantes reconozcan los cambios cualitativos en el desarrollo de habilidades de reflexión crítica. 
Harrison y Lee (2011) utilizando la misma rúbrica identificaron cambios en el nivel de práctica reflexiva y crítica entre los estudiantes, constatando avances en el desarrollo de la identidad profesional. Estos investigadores hicieron una adaptación para que se ajustará a los propósitos de su estudio. El estudio incluyó tanto las prácticas docentes del alumnado, como también otras categorías sobre su interacción con otros profesionales del contexto escolar.

Desde nuestro contexto en la modalidad dual, se valoró la necesidad de construir y validar un instrumento ad hoc para responder al escenario formativo particular de este estudio de caso. Así, desde una perspectiva multidimensional, se definieron 4 dimensiones que se despliegan en 8 categorías con 5 niveles de consecución. Las dimensiones Descripción, Comprensión, Proyección y Aspectos Formales y sus categorías orientan la escritura de los DFI, a la vez que sirven como herramienta para el análisis y la evaluación.

\section{Método}

El estudio explora la idoneidad de un instrumento correspondiente al Sistema de Categorías para el Análisis de Narrativas (SCAN) que evalúa y permite dar feedback al alumnado del GEP-dual sobre las narrativas que producen a partir de su experiencia educativa. Esta investigación se desarrolla como un estudio de caso, entendiendo como tal un análisis intensivo de una situación y su contexto, con la intención de comprender su evolución (Creswell, Hanson, Clark-Plano \& Morales, 2007).

Se trata de un diseño longitudinal cuasi-experimental que, mediante la construcción y validación de un instrumento, permite estudiar la evolución de las narrativas de los DFI entre dos puntos de control: al inicio del $2^{o}$ curso (pretest) y al final de $3 r$ curso (postest). Entre estos puntos de control se desarrolla una formación específica explicada posteriormente en el procedimiento.

Este proceso se ha llevado a cabo con tres cohortes consecutivas. En esta experiencia utilizamos el término cohorte como grupo de personas que se matricularon en 1r curso del GEP-dual el mismo año académico. Para minimizar las diferencias entre cohortes, todas recibieron la misma formación con el mismo profesorado, sesiones y contenidos.

\section{Objetivos}

Planteamos dos objetivos principales: a) construir y validar un instrumento para evaluar las narrativas de los DFI; b) analizar la evolución de las narrativas sobre la propia práctica educativa. Para ello, se definen cuatro objetivos específicos:

- Explorar la validez del contenido y la fiabilidad del instrumento.

- Analizar las relaciones entre las categorías de SCAN.

- Identificar el progreso de los DFI entre el prestest y el postest.

- Estudiar las diferencias y similitudes entre las cohortes. 


\section{Población y muestra}

La muestra intencional (Tabla 1) la conforman 150 DFI en el transcurso del $2^{\underline{0}}$ y 3r año del Grado de Educación Primaria. Provienen de tres cohortes consecutivas: 2014/2015, 2015/2016 y 2016/2017. Todos ellos participan de forma prescriptiva, según el plan de estudios de la modalidad dual, en la actividad docente de distintos centros escolares durante 2 días a la semana en $1^{\circ}, 2^{\underline{o}}$ y 3 r curso y con un Prácticum intensivo, de 3 meses y medio en $4^{\mathrm{o}}$ curso (Figura 1).

Tabla 1.

Características de la muestra.

\begin{tabular}{ccccc}
\hline Cohorte & № de DFI & Edad Media (DS) & Mujeres \% & Cursos analizados \\
\hline $2014-2015$ & 54 & $20.29(1.61)$ & 59.18 & $15 / 16,16 / 17$ \\
$2015-2016$ & 55 & $20.30(1.55)$ & 61.11 & $16 / 17,17 / 18$ \\
$2016-2017$ & 41 & $19.92(1.09)$ & 65.52 & $17 / 18,18 / 19$ \\
\hline & 150 & $20.16(1.42)$ & 62.10 & \\
\hline
\end{tabular}

\section{Instrumento}

SCAN (ver Apéndice) se diseñó con varios propósitos: conseguir una evaluación más objetiva, establecer criterios comunes entre el profesorado y favorecer procesos de autoevaluación y coevaluación de los DFI sobre sus propias narrativas. También para los mentores escolares que desde la función de acompañamiento asesoran a los DFI en sus observaciones, intervenciones, análisis y reflexiones. Su utilidad puede situarse en tres fases que contribuyen al desarrollo de la Visión Profesional de los DFI. En una fase preactiva con la planificación y estructuración de la narrativa, en una fase activa durante la redacción como guía y en una fase postactiva de evaluación (Sherin, 2007).

En la elaboración de las tres primeras dimensiones se tuvieron en cuenta las dimensiones descritas por Ward y McCotter (2004) adaptándolas para que conecten con el plan de estudios del GEP-Dual. Se definió una cuarta dimensión de carácter formal: expresión escrita y uso de la bibliografía, vinculada directamente con la movilización de los marcos teóricos. Para la definición de las categorías se tuvo en cuenta la aportación de Domingo y Gómez (2014) y su rúbrica ATOM para la práctica reflexiva.

Un grupo de diez expertos evaluó la validez del contenido del instrumento. La valoración de la experticia se basó en criterios de experiencia, implicación y participación activa en el GEP-dual. Para el criterio de experiencia se consideró que debían ser profesionales que ejerzan el rol de tutor académico, mentor escolar u otros profesionales con una docencia universitaria superior a 5 años. Se pidió a los expertos que evaluaran la pertinencia y la univocidad en la comprensión de los enunciados, donde solo se aceptaron los ítems que tenían más de un .80 de acuerdo. Tras un proceso iterativo con 
rondas sucesivas, la validez de contenido de la versión final del instrumento mediante la prueba V de Aiken resulto ser 83 (Salkind, 2016).

El SCAN tiene consistencia interna como nos indican los coeficientes obtenidos con el alpha de Cronbach, que son de .882 en el pretest y de .890 en el postest.

El instrumento (Tabla 2) sigue la estructura propuesta para la entrega de las narrativas y se compone de las siguientes 4 dimensiones:

- Descripción: presentación y contextualización de la situación educativa, el clima del aula, la actividad que se desarrolla, el diseño de la intervención, la metodología, los recursos y el desarrollo de la sesión. La contextualización facilita la comprensión de la situación educativa.

- Comprensión: reconstrucción y argumentación teórico-práctica. Cómo se explica, reflexiona y argumenta la intervención realizada movilizando marcos teóricos y aportando evidencias sobre la práctica.

- Proyección: interpretación crítica sobre la propia práctica para extraer los elementos más relevantes como puntos fuertes y débiles. Se reflexiona sobre las decisiones tomadas, las competencias movilizadas y posibles alternativas para futuras intervenciones.

- Aspectos formales: se atiende a la corrección lingüística y a la citación y referenciación de los marcos teóricos utilizados en las narrativas.

Tabla 2.

Resumen de las dimensiones y categorías del SCAN.

\begin{tabular}{cllllll}
\hline Dimensión & \multicolumn{1}{c}{ Categoría } & $\mathbf{1}$ & $\mathbf{2}$ & $\mathbf{3}$ & $\mathbf{4}$ & $\mathbf{5}$ \\
\hline Descripción & Presentación/ Contextualización & 1 & 2 & 3 & 4 & 5 \\
\hline Comprensión & Evidencias & 1 & 2 & 3 & 4 & 5 \\
& Argumentación & 1 & 2 & 3 & 4 & 5 \\
\hline \multirow{3}{*}{ Proyección } & Intervención Docente & 1 & 2 & 3 & 4 & 5 \\
& Competencias Docentes & 1 & 2 & 3 & 4 & 5 \\
& Oportunidades de Desarrollo & 1 & 2 & 3 & 4 & 5 \\
\hline \multirow{2}{*}{ Aspectos Formales } & Expresión Escrita & 1 & 2 & 3 & 4 & 5 \\
& Citación y Bibliografía & 1 & 2 & 3 & 4 & 5 \\
\hline
\end{tabular}

La evaluación de las narrativas considera en las distintas categorías 5 niveles de consecución. Los niveles 1,3 y 5 están descritos textualmente. Los niveles 2 y 4 no presentan descripción, esto permite hacer una valoración intermedia, siguiendo la propuesta de Good et al. (2006) en su Instrumento de Observación de Clases. 


\section{Procedimiento de recogida y análisis de datos}

En esta investigación se han tomado los valores de los análisis de las narrativas. Con las tres cohortes se siguieron las siguientes fases (Figura 2) establecidas en el diseño de la actividad:

- Fase 0. El profesorado presenta al alumnado la actividad de redacción de las narrativas que realizarán durante $2^{\circ}$ y 3 r curso. Se facilita un guion orientativo con la misma estructura y orden de las dimensiones de SCAN. Las narrativas solicitadas, de temática abierta, deben explicar y analizar las situaciones educativas experimentadas en la escuela.

En esta fase se realizan tres simulaciones de evaluación de narrativas realizadas por estudiantes de cursos anteriores, de las cuales se escogen intencionalmente referentes con evaluaciones altas y bajas. Así se proporcionan modelos para comprender y ensayar el uso de SCAN.

- Fase 1. Los estudiantes entregan la primera narrativa (pretest) que es evaluada mediante el SCAN. Se realiza una devolución de la evaluación mediante un seminario.

- Fase 2. Los estudiantes en distintas sesiones formativas a lo largo de $2^{\mathrm{o}}$ y $3 \mathrm{r}$ curso participan en debates, seminarios y presentaciones de docentes escolares con el fin de facilitar la integración entre la experiencia en el aula escolar con los marcos teóricos.

- Fase 3. Los estudiantes entregan la última narrativa (postest).

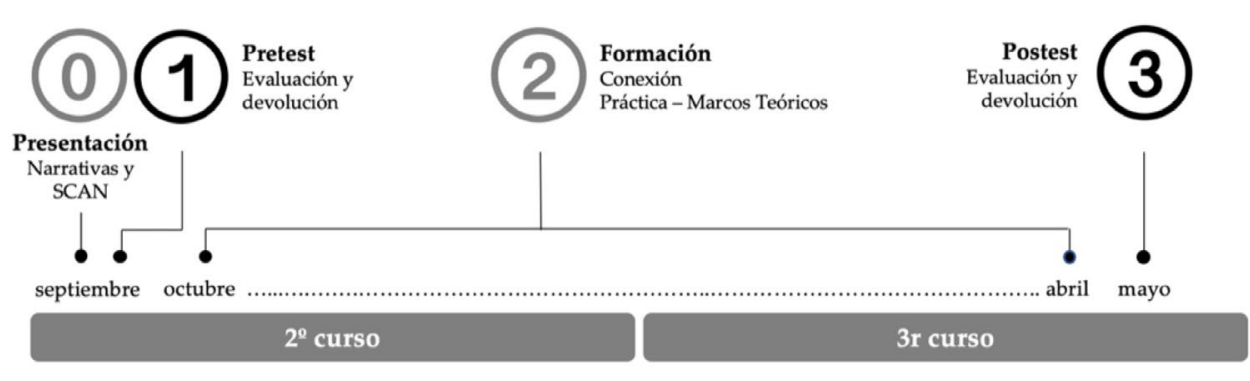

Figura 2. Fases del proceso de formación y recogida de datos.

\section{Resultados}

Para ver la evolución de los DFI, se compararon sus resultados en el pretest y el postest. Además, se confrontaron los resultados de las cohortes para comprobar si el progreso fue similar o no. Al no presentar una distribución normal $\left(D_{(150)}=.08, p=.012\right.$ para la puntuación del pretest y $D_{(150)}=.10, p<.001$ para el postest), los datos requieren pruebas no-paramétricas. 
Primero, se analizaron las relaciones entre las categorías de evaluación a través de correlaciones Spearman (Tabla 3 y 4 ).

Tabla 3.

Matriz de correlaciones Spearman en el pretest de las categorías del SCAN.

\begin{tabular}{|l|llllllll}
\cline { 2 - 8 } \multicolumn{1}{l}{} & PC & EV & AR & ID & CD & OD & EE & CB \\
\cline { 2 - 8 } EV & & & & & & & \\
AR & $.422^{*}$ & & & & & & \\
ID & $.532^{*}$ & $.335^{*}$ & & & & & \\
CD & $.555^{*}$ & $.571^{*}$ & $.459^{*}$ & & & & \\
OD & $.586^{*}$ & $.530^{*}$ & $.499^{*}$ & $.681^{*}$ & & & \\
EE & $.624^{*}$ & $.435^{*}$ & $.572^{*}$ & $.720^{*}$ & $.721^{*}$ & & \\
CB & $.412^{*}$ & $.277^{*}$ & $.513^{*}$ & $.309^{*}$ & $.358^{*}$ & $.361^{*}$ & \\
& $.555^{*}$ & $.426^{*}$ & $.521^{*}$ & $.483^{*}$ & $.587^{*}$ & $.477^{*}$ & $.395^{*}$ \\
\hline
\end{tabular}

${ }^{*} p<.05$

Tabla 4.

Matriz de correlaciones Spearman en el postest de las categorías del SCAN.

\begin{tabular}{|l|llllllll}
\cline { 2 - 8 } PC & PC & EV & AR & ID & CD & OD & EE & CB \\
\cline { 2 - 8 } EV & & & & & & & \\
AR & $.507^{*}$ & & & & & & \\
ID & $.548^{*}$ & $.326^{*}$ & & & & & \\
CD & $.587^{*}$ & $.622^{*}$ & $.408^{*}$ & & & & \\
OD & $.502^{*}$ & $.482^{*}$ & $.517^{*}$ & $.530^{*}$ & & & \\
EE & $.678^{*}$ & $.565^{*}$ & $.531^{*}$ & $.638^{*}$ & $.689^{*}$ & & \\
CB & $.496^{*}$ & .128 & $.571^{*}$ & $.345^{*}$ & $.380^{*}$ & $.404^{*}$ & \\
& $.515^{*}$ & $.379^{*}$ & $.716^{*}$ & $.388^{*}$ & $.608^{*}$ & $.517^{*}$ & $.536^{*}$ \\
\hline
\end{tabular}

${ }^{*} p<.05$

En la matriz de correlaciones destaca que cuando los estudiantes del GEP-dual mejoran en una categoría también lo hacen en las demás. Este progreso es consistente y se relaciona en todas las categorías del SCAN. Específicamente, se destacan las altas correlaciones entre Oportunidades de Desarrollo con Intervención Docente, Competencias Docentes y con Presentación y Contextualización. Se distingue también una correlación 
moderada en el pretest, que evoluciona a alta en el postest, entre las categorías de Argumentación y Citación y Bibliografía.

En segundo lugar, se compararon los resultados del pretest y del postest para cada una de las categorías de SCAN, primero para cada una de las tres cohortes y segundo para toda la muestra. Los resultados se presentan en la Tabla 5, que incluye los estadísticos descriptivos, los resultados de las pruebas de Wilcoxon realizadas con este fin y los tamaños de efecto correspondientes.

A nivel general, las desviaciones estándar sugieren una ligera disminución en la variabilidad de los resultados de los DFI. Los resultados de las pruebas de Wilcoxon muestran que todas las cohortes obtuvieron puntuaciones más altas en el Postest que en el pretest con respecto a Presentación y Contextualización, Evidencias, Argumentación, Intervención Docente, Competencias Docentes, Oportunidades de Desarrollo, Expresión Escrita y Citación y Bibliografía. En otras palabras, los participantes mejoraron en todas las categorías del sistema SCAN. Además, considerando los grandes tamaños de efecto obtenidos, esta mejora tiene un significado práctico. El progreso hecho por los DFI es destacable en las categorías Intervención Docente, Competencias Docentes y Oportunidades de Desarrollo.

Tabla 5.

Estadísticos descriptivos y prueba Wilcoxon del pretest y postest por cohortes.

\begin{tabular}{|c|c|c|c|c|c|c|c|}
\hline Categoría evaluación & Cohorte & Narrativa & Mediana & Media & DS & $\begin{array}{c}\text { Prueba } \\
\text { Wilcoxon }\end{array}$ & $\begin{array}{c}\text { Tamaño } \\
\text { efecto }\end{array}$ \\
\hline \multirow{8}{*}{$\begin{array}{l}\text { Presentación } \\
\text { Contextualización } \\
\text { (PC) }\end{array}$} & \multirow{2}{*}{$14-15$} & Pretest & 3 & 3.06 & .86 & $Z=5.69$ & \multirow{2}{*}{.77} \\
\hline & & Postest & 4 & 4.04 & .85 & $p<.001$ & \\
\hline & \multirow{2}{*}{$15-16$} & Pretest & 4 & 3.38 & 1.10 & $Z=5.66$ & \multirow{2}{*}{.75} \\
\hline & & Postest & 5 & 4.53 & .63 & $p<.001$ & \\
\hline & \multirow{2}{*}{$16-17$} & Pretest & 3 & 3.39 & .95 & $Z=4.05$ & \multirow{2}{*}{.63} \\
\hline & & Postest & 4 & 4.12 & .93 & $p<.001$ & \\
\hline & \multirow{2}{*}{ Total } & Pretest & 3 & 3.27 & .98 & $Z=8.89$ & \multirow{2}{*}{.73} \\
\hline & & Postest & 4 & 4.24 & .83 & $p<.001$ & \\
\hline \multirow{8}{*}{$\begin{array}{l}\text { Evidencias } \\
\text { (EV) }\end{array}$} & \multirow{2}{*}{$14-15$} & Pretest & 3 & 2.87 & 1.32 & $Z=5.33$ & \multirow{2}{*}{.73} \\
\hline & & Postest & 5 & 4.28 & 1.02 & $p<.001$ & \\
\hline & \multirow{2}{*}{$15-16$} & Pretest & 3 & 2.64 & 1.60 & $Z=4.63$ & \multirow{2}{*}{.62} \\
\hline & & Postest & 5 & 4.11 & 1.36 & $p<.001$ & \\
\hline & \multirow{2}{*}{$16-17$} & Pretest & 2 & 2.44 & 1.48 & $Z=4.56$ & \multirow{2}{*}{.71} \\
\hline & & Postest & 4 & 3.76 & 1.32 & $p<.001$ & \\
\hline & \multirow{2}{*}{ Total } & Pretest & 3 & 2.67 & 1.47 & $Z=8.33$ & \multirow{2}{*}{.68} \\
\hline & & Postest & 5 & 4.07 & 1.24 & $p<.001$ & \\
\hline
\end{tabular}




\begin{tabular}{|c|c|c|c|c|c|c|c|}
\hline Categoría evaluación & Cohorte & Narrativa & Mediana & Media & DS & $\begin{array}{l}\text { Prueba } \\
\text { Wilcoxon }\end{array}$ & $\begin{array}{c}\text { Tamaño } \\
\text { efecto }\end{array}$ \\
\hline \multirow{8}{*}{ Argumentación (AR) } & \multirow{2}{*}{$14-15$} & Pretest & 3 & 2.91 & .94 & $Z=4.50$ & \multirow{2}{*}{.61} \\
\hline & & Postest & 4 & 3.56 & .86 & $p<.001$ & \\
\hline & \multirow{2}{*}{$15-16$} & Pretest & 3 & 3.11 & 1.06 & $Z=5.54$ & \multirow{2}{*}{.75} \\
\hline & & Postest & 4 & 4.00 & .77 & $p<.001$ & \\
\hline & \multirow{2}{*}{$16-17$} & Pretest & 3 & 3.10 & .97 & $Z=4.13$ & \multirow{2}{*}{.64} \\
\hline & & Postest & 4 & 3.78 & .85 & $p<.001$ & \\
\hline & \multirow{2}{*}{ Total } & Pretest & 3 & 3.03 & .98 & $Z=8.21$ & \multirow{2}{*}{.67} \\
\hline & & Postest & 4 & 3.78 & .84 & $p<.001$ & \\
\hline \multirow{8}{*}{$\begin{array}{l}\text { Intervención } \\
\text { Docente } \\
\text { (ID) }\end{array}$} & \multirow{2}{*}{$14-15$} & Pretest & 3.50 & 3.44 & .86 & $Z=4.72$ & \multirow{2}{*}{.64} \\
\hline & & Postest & 4 & 4.17 & .84 & $p<.001$ & \\
\hline & \multirow{2}{*}{$15-16$} & Pretest & 3 & 3.18 & 1.06 & $Z=5.64$ & \multirow{2}{*}{.76} \\
\hline & & Postest & 5 & 4.35 & .82 & $p<.001$ & \\
\hline & \multirow{2}{*}{$16-17$} & Pretest & 3 & 3.00 & 1.00 & $Z=4.84$ & \multirow{2}{*}{.76} \\
\hline & & Postest & 4 & 3.93 & .85 & $p<.001$ & \\
\hline & \multirow{2}{*}{ Total } & Pretest & 3 & 3.23 & .98 & $Z=8.74$ & \multirow{2}{*}{.71} \\
\hline & & Postest & 4 & 4.17 & .85 & $p<.001$ & \\
\hline \multirow{8}{*}{$\begin{array}{l}\text { Competencias } \\
\text { Docentes } \\
\text { (CD) }\end{array}$} & \multirow{2}{*}{$14-15$} & Pretest & 2 & 2.37 & 1.05 & $Z=5.05$ & \multirow{2}{*}{.69} \\
\hline & & Postest & 4 & 3.35 & 1.08 & $p<.001$ & \\
\hline & \multirow{2}{*}{$15-16$} & Pretest & 2 & 2.15 & 1.18 & $Z=5.63$ & \multirow{2}{*}{.76} \\
\hline & & Postest & 4 & 3.65 & 1.25 & $p<.001$ & \\
\hline & \multirow{2}{*}{$16-17$} & Pretest & 2 & 2.12 & 1.08 & $Z=4.55$ & \multirow{2}{*}{.71} \\
\hline & & Postest & 4 & 3.39 & 1.12 & $p<.001$ & \\
\hline & \multirow{2}{*}{ Total } & Pretest & 2 & 2.22 & 1.10 & $Z=8.76$ & \multirow{2}{*}{.71} \\
\hline & & Postest & 4 & 3.47 & 1.16 & $p<.001$ & \\
\hline \multirow{8}{*}{$\begin{array}{l}\text { Oportunidades de } \\
\text { Desarrollo (OD) }\end{array}$} & \multirow{2}{*}{$14-15$} & Pretest & 3 & 2.81 & 1.05 & $Z=5.57$ & 76 \\
\hline & & Postest & 4 & 3.78 & .95 & $p<.001$ & .70 \\
\hline & 15 & Pretest & 3 & 2.62 & 1.18 & $Z=5.91$ & ( \\
\hline & $15-10$ & Postest & 4 & 3.95 & 1.18 & $p<.001$ & .80 \\
\hline & 1617 & Pretest & 3 & 2.56 & 1.18 & $Z=5.10$ & \\
\hline & $10-1$ & Postest & 4 & 3.76 & .99 & $p<.001$ & .00 \\
\hline & To & Pretest & 3 & 2.67 & 1.13 & $Z=9.54$ & 70 \\
\hline & Iolat & Postest & 4 & 3.83 & 1.05 & $p<.001$ & .70 \\
\hline
\end{tabular}




\begin{tabular}{|c|c|c|c|c|c|c|c|}
\hline Categoría evaluación & Cohorte & Narrativa & Mediana & Media & DS & $\begin{array}{l}\text { Prueba } \\
\text { Wilcoxon }\end{array}$ & $\begin{array}{c}\text { Tamaño } \\
\text { efecto }\end{array}$ \\
\hline \multirow{8}{*}{$\begin{array}{l}\text { Expresión Escrita } \\
\text { (EE) }\end{array}$} & \multirow{2}{*}{$14-15$} & Pretest & 3 & 3.07 & .93 & $Z=4.64$ & \multirow{2}{*}{.63} \\
\hline & & Postest & 4 & 3.59 & .81 & $p<.001$ & \\
\hline & \multirow{2}{*}{$15-16$} & Pretest & 4 & 3.60 & .96 & $Z=4.19$ & \multirow{2}{*}{.57} \\
\hline & & Postest & 4 & 4.25 & .78 & $p<.001$ & \\
\hline & \multirow{2}{*}{$16-17$} & Pretest & 3 & 3.32 & .93 & $Z=3.62$ & \multirow{2}{*}{.57} \\
\hline & & Postest & 4 & 3.93 & .82 & $p<.001$ & \\
\hline & \multirow{2}{*}{ Total } & Pretest & 3 & 3.33 & .96 & $Z=7.12$ & \multirow{2}{*}{.58} \\
\hline & & Postest & 4 & 3.93 & .84 & $p<.001$ & \\
\hline \multirow{8}{*}{$\begin{array}{c}\text { Citación y } \\
\text { Bibliografía (CB) }\end{array}$} & \multirow{2}{*}{$14-15$} & Pretest & 3 & 2.65 & .94 & $Z=4.45$ & \multirow{2}{*}{.60} \\
\hline & & Postest & 3 & 3.28 & .94 & $p<.001$ & \\
\hline & \multirow{2}{*}{$15-16$} & Pretest & 3 & 2.69 & 1.15 & $Z=5.03$ & \multirow{2}{*}{.68} \\
\hline & & Postest & 4 & 3.73 & 1.25 & $p<.001$ & \\
\hline & \multirow{2}{*}{$16-17$} & Pretest & 3 & 2.93 & 1.19 & $Z=2.94$ & \multirow{2}{*}{.46} \\
\hline & & Postest & 4 & 3.49 & 1.17 & $p<.001$ & \\
\hline & \multirow{2}{*}{ Total } & Pretest & 3 & 2.74 & 1.09 & $Z=7.27$ & \multirow{2}{*}{.59} \\
\hline & & Postest & 4 & 3.50 & 1.13 & $p<.001$ & \\
\hline \multirow{8}{*}{ Puntuación total } & \multirow{2}{*}{$14-15$} & Pretest & 2.81 & 2.90 & .76 & $Z=6.40$ & \multirow{2}{*}{.87} \\
\hline & & Postest & 3.81 & 3.75 & .72 & $p<.001$ & \\
\hline & \multirow{2}{*}{$15-16$} & Pretest & 2.88 & 2.92 & .85 & $Z=6.46$ & \multirow{2}{*}{.87} \\
\hline & & Postest & 4.38 & 4.07 & .74 & $p<.001$ & \\
\hline & \multirow{2}{*}{$16-17$} & Pretest & 2.88 & 2.86 & .83 & $Z=5.59$ & \multirow{2}{*}{.87} \\
\hline & & Postest & 3.88 & 3.77 & .78 & $p<.001$ & \\
\hline & \multirow{2}{*}{ Total } & Pretest & 2.88 & 2.90 & .81 & $Z=10.63$ & \multirow{2}{*}{.87} \\
\hline & & Postest & 4 & 3.87 & .76 & $p<.001$ & \\
\hline
\end{tabular}

Tercero, se compararon las cohortes usando la prueba Kruskal-Wallis. Los resultados indican un punto de partida similar de las tres cohortes, sin diferencias significativas en el pretest $H_{(2)}=.19, p=.907$. La evolución en las puntuaciones fue mayor en la cohorte 15-16. La prueba Kruskal-Wallis muestra una mejora de las puntuaciones de esta cohorte, con resultados significativamente más altos que los de la anterior, 14-15 $(p=.29, r=.25)$. No se identifican diferencias estadísticamente significativas entre las cohortes $14-15$ y 16-17 ( $p=1.000, r=.05)$ y entre las cohortes 15-16 y 16-17 ( $p=.106, r=.21)$. 
Tabla 6.

Prueba de Kruskal-Wallis del pretest y postest.

\begin{tabular}{|c|c|c|c|c|c|c|c|}
\hline & Categoría & $\begin{array}{l}\text { Kruskal- } \\
\text { Wallis }\end{array}$ & $p$ & & Categoría & $\begin{array}{l}\text { Kruskal- } \\
\text { Wallis }\end{array}$ & $p$ \\
\hline \multirow{8}{*}{ Pretest } & PC & 4.82 & .090 & \multirow{8}{*}{ Postest } & PC & 10.49 & .005 \\
\hline & EV & 1.92 & .383 & & EV & 4.57 & .102 \\
\hline & $\mathrm{AR}$ & 2.15 & .341 & & AR & 7.35 & .025 \\
\hline & ID & 5.28 & .071 & & ID & 6.89 & .032 \\
\hline & $\mathrm{CD}$ & 2.17 & .338 & & $\mathrm{CD}$ & 2.72 & .256 \\
\hline & OD & 1.02 & .601 & & OD & 2.53 & .283 \\
\hline & EE & 10.75 & .005 & & EE & 18.86 & .000 \\
\hline & $\mathrm{CB}$ & 2.18 & .336 & & $\mathrm{CB}$ & 7.83 & .020 \\
\hline
\end{tabular}

En el estudio los resultados indican que la cohorte 15-16 obtuvo mejores resultados que la cohorte 14-15 (Tabla 6). Con respecto a la categoría de Expresión Escrita con valores de $p=.001, r=.31$ en el pretest y $p<.001, r=.42$ en el postest. El mismo patrón se observa entre estas mismas cohortes en el postest, en la Presentación y Contextualización ( $p=.005$, $r=.30$ ), en Argumentación ( $p=.020, r=.26$ ) y en Citación y Bibliografía ( $p=.016, r=.27)$. Por otro lado, los estudiantes de la cohorte 16-17 obtuvieron mejores resultados que los de la cohorte 15-16 ( $p=.026, r=.27)$ en la categoría de Intervención Docente en el postest.

\section{Discusión y conclusiones}

Con relación al primer objetivo principal que nos planteamos, SCAN ha alcanzado una validez de contenido elevada con la participación de expertos y, por otro lado, el pilotaje con el alumnado nos ha permitido constatar su consistencia interna. Consecuentemente, este estudio ha permitido construir y validar un instrumento ad hoc que proporciona una evaluación más objetiva de las narrativas.

Asimismo, consideramos que todo el proceso formativo cumple con el segundo objetivo de analizar la evolución en términos de eficiencia. Se constata la mejoría de los DFI gracias a SCAN, que los guía en el proceso de integración teórico-práctico. Consideramos que la mejora proviene del propio proceso formativo, en concreto, de los seminarios de integración y el seguimiento de las narrativas con un feedback constante en la tutorización (fase 1 y 2).

Así, el proceso formativo favorece el desarrollo de la Visión Profesional con una interpretación más comprensiva de las experiencias educativas (Stürmer, Könings \& Seidel, 2014). SCAN permite identificar el cambio en las narrativas desde la descripción simple y la explicación influenciada por creencias personales hasta una argumentación que utiliza principios teóricos de forma contextualizada. La mejora puede deberse a la movilización de los marcos teóricos para interpretar la experiencia del aula, a la vez que la acción docente favorece la autocrítica. Las competencias docentes se sitúan en el centro del 
análisis promoviendo replanteamientos metodológicos sobre la propia intervención docente y la explicitación de necesidades formativas para resolver una situación real.

La necesidad de instrumentar la evaluación queda resuelta con el SCAN, ya que forma parte de la tutorización, proporcionando información al tutor académico, al mentor escolar y al DFI (Clerici \& Da Re, 2019). Además, esta información permite establecer el enfoque de la evaluación como un elemento formativo, mediante la autoevaluación, coevaluación entre iguales y heteroevaluación por parte del tutor académico y del mentor escolar (Good et al., 2006).

En primer lugar, la correlación entre categorías se evidencia tanto en el pretest como en el postest (Tabla 3 y 4). En la dimensión Proyección, destaca, una correlación elevada entre las categorías Intervención Docente y Competencias Docentes con la categoría Oportunidades de Desarrollo. Los DFI mejoran en la puntuación en Oportunidades de Desarrollo cuando argumentan con coherencia sus logros y plantean vías formativas para las necesidades de mejora de sus competencias profesionales. La formación entre el pretest y postest, con las narrativas y el SCAN, orienta esta mejora promoviendo el desarrollo de la identidad profesional y la apropiación de las competencias docentes (Correa-Molina, 2015). Fernández-Díaz, Rodríguez-Mantilla y Fernández-Cruz (2016) consideran que las dinámicas continuadas de reflexión promueven "la detección de necesidades de formación, teniendo en cuenta las competencias profesionales necesarias para el desempeño de su trabajo" (p. 87).

De igual modo, la categoría Oportunidad de Desarrollo presenta una correlación generalmente elevada con la categoría Presentación y Contextualización. Las propuestas de mejora que los DFI redactan como Oportunidades de Desarrollo tienen coherencia en el contexto donde se deberían implementar y, por consiguiente, con la descripción del aula, de su diversidad y complejidad, de las relaciones interpersonales y de las actividades de la sesión analizada en la narrativa. Consideramos que esta correlación es elevada por la participación activa de los DFI en la vida escolar durante todo el plan de estudios.

La categoría Argumentación mantiene una correlación moderada en el pretest, que evoluciona a alta en el postest con la categoría Citación y Bibliografía. Se han descrito ampliamente las dificultades que los DFI muestran para comprender e integrar los marcos teóricos durante su formación (Brante, Holmqvist, Holmquist \& Palla, 2015). Gracias a las sesiones de formación (fase 2) sobre revisión y lectura de textos los DFI mejoran de forma clara la argumentación sobre la práctica y la intención educativa, más allá de la opinión personal e ideas preestablecidas.

En segundo lugar, se observa en las puntuaciones globales en el pretest y el postest que existen diferencias estadísticamente significativas en las tres cohortes estudiadas. El tamaño del efecto es mayor en las tres primeras dimensiones que en la dimensión de Aspectos formales. Esto puede explicarse considerando que las primeras son más novedosas para los DFI y, además, el hecho de que tengan distintos mentores escolares puede producir cambios en su reflexión crítica sobre la práctica educativa. Mientras que la atención sobre la corrección lingüística y en la citación bibliográfica viene trabajándose a lo largo de toda su formación anterior y en este proceso todos reciben los mismos seminarios de integración. 
Al comparar el pretest con el postest mediante la prueba Wilcoxon (Tabla 5), en el postest se observan mejores resultados tanto en la puntuación total, como en cada una de las categorías de análisis del SCAN. El tamaño del efecto es grande en las tres cohortes ya que todas participan en el mismo proceso formativo detallado anteriormente. Estas diferencias estadísticamente significativas permiten constatar el progreso de los DFI a lo largo de dos cursos, entre los dos puntos de control, en sus narrativas.

En tercer lugar, las diferencias en los resultados de las tres las cohortes, aunque el procedimiento formativo fue similar, pueden explicarse por otras variables contextuales. Distintos estudios han considerado anteriormente diferencias en la eficiencia de distintos grupos (Warning, 2004). En relación con la diferencia de resultados, podemos suponer como variables extrañas de nuestro estudio el rendimiento académico de los componentes de las cohortes, la formación previa, aspectos de motivación e implicación en la propia formación, las reacciones del mismo profesorado universitario en los seminarios de integración, entre otras. López-Martín y González-Villanueva (2018) destacan la figura del tutor académico ya que "es importante profundizar en cómo influye el perfil del tutor, su motivación, formación y forma de relacionarse en el entorno de la tutoría" (p. 384).

Una posible limitación del SCAN es cómo su uso condiciona la explicación y argumentación sobre la experiencia práctica. Así, el estudiante estructura la narrativa en base a las dimensiones y categorías establecidas. Los resultados obtenidos con su uso podrían responder a las expectativas de los formadores más que a una verdadera evolución de la capacidad reflexiva (Beauchamp, 2015). En este sentido, constatamos la necesidad de alimentar distintas perspectivas de análisis para promover la autoconfrontación y la objetividad.

Para dar continuidad al estudio sobre el dispositivo formativo será necesario establecer una investigación experimental en modalidad dual, con un grupo que no utilice la herramienta SCAN. Este nuevo diseño experimental podría arrojar luz sobre las aportaciones más específicas de este instrumento. Por otro lado, otra posibilidad es implementar un estudio experimental incluyendo un grupo control que no realice su formación en modalidad dual pero que sí utilice el SCAN. No se dispone de datos que permitan conocer cómo esta variable condiciona a los DFI con relación a su reflexividad y práctica profesional. Debe tenerse en cuenta que una formación dual de calidad, además de ofrecer una mayor experiencia profesional, garantiza la interacción de los aprendizajes de ambos escenarios.

\section{Referencias}

Alsina, Á., Ayllón, S., Colomer, J., Fernández-Peña, R., Fullana, J., Pallisera, M., ... \& Serra, L. (2017). Improving and evaluating reflective narratives: A rubric for higher education students. Teaching and Teacher Education, 63(3), 148-158. doi: 10.1016/j. tate.2016.12.015

Álvarez-Santullano, M., \& De Prada, E. (2018). Evaluación de las competencias profesionales a través de las prácticas externas: incidencia de la creatividad. Revista de Investigación Educativa, 36(1), 203-219. doi: 10.6018/rie.36.1.275651 
Beauchamp, C. (2015). Reflection in teacher education: issues emerging from a review of current literature. Reflective Practice, 16(1), 123-141. doi: 10.1080/14623943.2014.982525

Brante, G., Holmqvist, M., Holmquist, P., \& Palla, M. (2015). Theorising teaching and learning: pre-service teachers' theoretical awareness of learning. European Journal of Teacher Education, 38(1), 102-118. doi: 10.1080/02619768.2014.902437

Bucheton, D., \& Soulé, Y. (2009). Les gestes professionnels et le jeu des postures de l'enseignant dans la classe: un multi-agenda de préoccupations enchâssées. Éducation et didactique, 3(3), 29-48. doi: 10.4000/educationdidactique.543

Carandell, Z., Keim, L., \& Tigchelaar, A. (2010). Herramientas para fomentar procesos de autorregulación. En O. Esteve, K. Melief, \& A. Alsina (Eds.), Creando mi profesión. Una propuesta para el desarrollo profesional del profesorado (pp. 65-96). Barcelona: Editorial Octaedro.

Clerici, R., \& Da Re, L. (2019). Evaluación de la eficacia de un programa de tutoría formativa. Revista de Investigación Educativa, 37(1), 39-56. doi: 10.6018/rie.37.1.32233

Coiduras, J., Correa-Molina, E., Boudjaoui, M., \& Curto, A. (2017). Formación dual en el grado de educación: claves organizativas y pedagógicas. Revista Qurriculum, 30, 81-102. Recuperado de http://riull.ull.es/xmlui/handle/915/6138

Correa-Molina, E. (2015). La alternancia en la formación inicial docente. Educar, 51(2), 259-279. doi: 10.5565/rev/educar.712

Creswell, J. W., Hanson, W. E., Clark-Plano, V. L., \& Morales, A. (2007). Qualitative research designs: Selection and implementation. The counseling psychologist, 35(2), 236-264. doi: 10.1177/0011000006287390

Danielson, C. (2013). The Framework For Teaching: evaluation instrument. Princeton: The Danielson Group.

Domingo, À. \& Gómez, V. (2014). La práctica reflexiva. Bases, modelos e instrumentos. Madrid: Narcea Ediciones.

Fernández-Díaz, M. J., Rodríguez-Mantilla, J. M., \& Fernández-Cruz, F. J. (2016). Evaluación de competencias docentes del profesorado para la detección de necesidades formativas. Bordón. Revista de pedagogía, 68(2), 85-101. doi: 10.13042/Bordon.2016.68206

Ferrández-Berrueco, R., \& Sánchez-Zarazaga, L. (2019). Las prácticas externas desde la perspectiva de las entidades colaboradoras. RELIEVE, 25(1), art. 5., 1-14. doi: 10.7203/relieve.25.1.13189

Good, T. L., McCaslin, M., Tsang, H. Y., Zhang, J., Wiley, C. R. H., Bozack, A. R., \& Hester, W. (2006). How well do 1st-year teachers teach does type of make a difference? Journal of Teacher Education, 57(4), 410-430. doi: 10.1177/0022487106291566

Goyette, N., \& Martineau, S. (2018). Les défis de la formation initiale des enseignants et le développement d'une identité professionnelle favorisant le bien-être. Phronesis, 7(4), 4-19. doi: 10.7202/1056316ar

Hanna, F., Oostdam, R., Severiens, S. E., \& Zijlstra, B. J. (2020). Assessing the professional identity of primary student teachers: Design and validation of the Teacher Identity Measurement Scale. Studies in Educational Evaluation, 64, 1-10. doi: 10.1016/j. stueduc.2019.100822

Harrison, J. K., \& Lee, R. (2011). Exploring the use of critical incident analysis and the professional learning conversation in an initial teacher education programme. Journal of Education for Teaching, 37(2), 199-217. doi: 10.1080/02607476.2011.558285 
Korthagen, F. (2017). A foundation for effective teacher education: Teacher education pedagogybased on situated learning. En D. J. Clandinin, \& J. Husu (Eds.), The Sage handbook of research on teacher education (pp. 528-544). London: Sage Publications. doi: 10.4135/9781526402042.n30

Le Boterf, G. (2002). Développer la compétence des professionnels. (4 ed.) Paris: Éditions d'Organisation.

Leroux, M., \& Portelance, L. (2018). Les initiatives du milieu universitaire et du milieu scolaire pour favoriser la préparation à la transition vers l'insertion dans la profession enseignante. En P. Chaubet, M. Leroux, C., Masson, C., Gervais, \& A. Malo, (Eds), Apprendre et enseigner en contexte d'alternance: vers la définition d'un noyau conceptuel (pp. 247-273). Québec: Presses de l’Université du Québec.

López-Martín, I., \& González-Villanueva, P. (2018). La tutoría universitaria como espacio de relación personal. Un estudio de caso múltiple. Revista de Investigación Educativa, 36(2), 381-399. doi: 10.6018/rie.36.2.291161

Melief, K., Tigchelaar, A., Korthagen, F., \& van Rijswijk, M. (2010). Aprender de la práctica. En O. Esteve, K. Melief, \& A. Alsina (Eds.), Creando mi profesión. Una propuesta para el desarrollo profesional del profesorado (pp.19-38). Barcelona: Octaedro.

Munthe, E., \& Rogne, M. (2015). Research based teacher education. Teaching and Teacher Education, 46(2), 17-24. doi: 10.1016/j.tate.2014.10.006

Podolskiy, A. (2019). On the Way of Developing a Holistic Explanatory Model of Positive Learning. En O. Zlatkin-Troitschanskaia, (Eds.), Frontiers and Advances in Positive Learning in the Age of InformaTiOn (PLATO) (pp. 269-276). Cham: Springer. doi: 10.1007/978-3-030-26578-6_19

Salkind, N.J. (2016). Exploring Research (9 ed.). New Jersey: Pearson International Education.

Sarceda-Gorgoso, M.C., \& Rodicio-García, M. (2017). Escenarios formativos y competencias profesionales en la formación inicial del profesorado. Revista Complutense de Educación, 29(1), 147-163. doi: 10.5209/RCED.52160

Seidel, T., \& Stürmer, K. (2014). Modeling and measuring the structure of professional vision in preservice teachers. American Educational Research Journal, 51(4), 739-77. doi: 10.3102/0002831214531321

Sherin, M. G. (2007). The development of teachers' professional vision in video clubs. En R. Goldman, R. Pea, B. Barron, \& S. J. Derry (Eds.), Video research in the learning sciences (pp. 383-395). New Jersey: Lawrence Erlbaum.

Stürmer, K., Könings, K.D., \& Seidel, T. (2014). Factors within University-Based Teacher Education relating to preservice teachers' professional vision. Vocations and Learning, 8(1), 35-54. doi: 10.1007/s12186-014-9122-z

van Es, E. A., Cashen, M., Barnhart, T., \& Auger, A. (2017). Learning to notice mathematics instruction: Using video to develop preservice teachers' vision of ambitious pedagogy. Cognition and Instruction, 35(3), 165-187. doi: 10.1080/07370008.2017.1317125

Ward, J. R., \& McCotter, S. S. (2004). Reflection as a visible outcome for preservice teachers. Teaching and Teacher Education, 20(3), 243-257. doi: 10.1016/j.tate.2004.02.004

Warning, S. (2004). Performance Differences in German Higher Education: Empirical Analysis of Strategic Groups. Review of Industrial Organization, 24(4), 393-408. doi: 10.1023/B:REIO.0000037538.48594.2c 
Watts, M., \& Lawson, M. (2009). Using a meta-analysis activity to make critical reflection explicit in teacher education. Teaching and Teacher Education, 25(5), 609-616. doi: 10.1016/j.tate.2008.11.019

Fecha de recepción: 19 de febrero de 2020.

Fecha de revisión: 9 de marzo de 2020.

Fecha de aceptación: 12 de junio de 2020. 


\section{Apéndice}

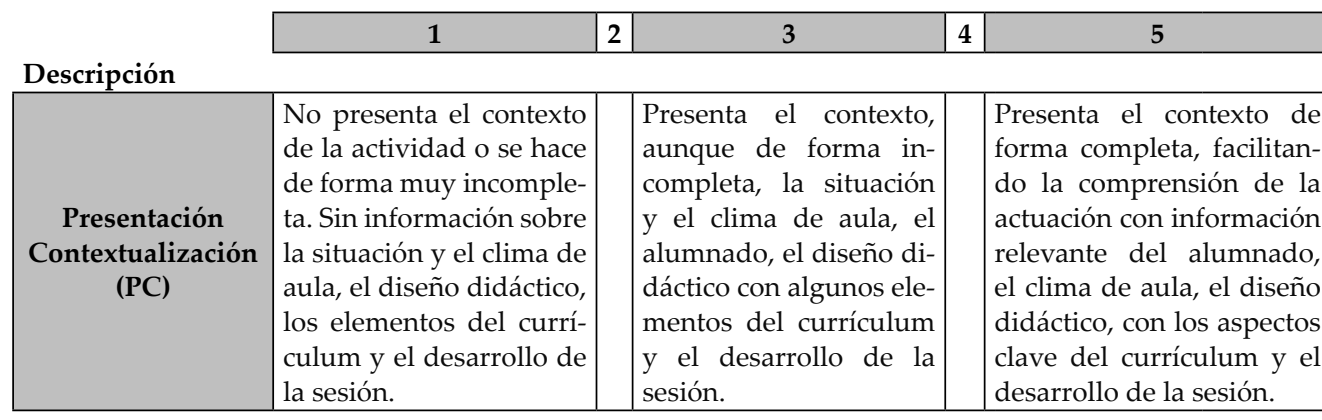

\section{Comprensión}

\begin{tabular}{|c|c|c|c|}
\hline $\begin{array}{l}\text { Evidencias } \\
\text { (EV) }\end{array}$ & $\begin{array}{l}\text { No documenta la inter- } \\
\text { vención, o lo hace de for- } \\
\text { ma insuficiente, con: re- } \\
\text { cursos, planificaciones, } \\
\text { producciones del alum- } \\
\text { nado, elaboración de } \\
\text { materiales, informes de } \\
\text { evaluación... o de otros } \\
\text { elementos relevantes. }\end{array}$ & $\begin{array}{l}\text { Documenta parcial- } \\
\text { mente la intervención } \\
\text { con información rele- } \\
\text { vante sobre: recursos, } \\
\text { planificaciones, pro- } \\
\text { ducciones del alum- } \\
\text { nado, elaboración de } \\
\text { materiales, informes de } \\
\text { evaluación... estableci- } \\
\text { endo una relación par- } \\
\text { cial entre la narrativa y } \\
\text { las evidencias. }\end{array}$ & $\begin{array}{l}\text { Documenta la situación vi- } \\
\text { vida con información rele- } \\
\text { vante sobre: recursos, pla- } \\
\text { nificaciones, producciones } \\
\text { del alumnado, elaboración } \\
\text { de materiales, informes } \\
\text { de evaluación... estableci- } \\
\text { endo narrativamente una } \\
\text { conexión consistente y } \\
\text { pertinente. }\end{array}$ \\
\hline \multirow[t]{2}{*}{$\begin{array}{l}\text { Argumentación } \\
\text { (AR) }\end{array}$} & $\begin{array}{l}\text { El discurso se sitúa en el } \\
\text { sentido común, en per- } \\
\text { cepciones o emociones, } \\
\text { o en la mera descripción. } \\
\text { Sin relación, o débil, con } \\
\text { los marcos teóricos (sin } \\
\text { autores). Se dan afirma- } \\
\text { ciones gratuitas, super- } \\
\text { fluas o se expresan pre- } \\
\text { juicios. }\end{array}$ & $\begin{array}{l}\text { El discurso sobre la } \\
\text { experiencia se apoya } \\
\text { en marcos teóricos con } \\
\text { conceptos, teorías y } \\
\text { autores y/o en otras } \\
\text { experiencias docentes. } \\
\text { Las temáticas e inter- } \\
\text { pretaciones son parci- } \\
\text { almente pertinentes y } \\
\text { relevantes. }\end{array}$ & $\begin{array}{l}\text { Se confronta y explica la } \\
\text { práctica con los marcos } \\
\text { teóricos, movilizando con- } \\
\text { ceptos, teorías y autores } \\
\text { y/o con otras experiencias } \\
\text { docentes de forma per- } \\
\text { tinente y relevante. Pre- } \\
\text { senta nuevos puntos de } \\
\text { vista. }\end{array}$ \\
\hline & 1 & 2 & 4 \\
\hline
\end{tabular}

\section{Proyección}

\begin{tabular}{|c|l|l|l|l|}
\hline $\begin{array}{c}\text { Explica la actuación des- } \\
\text { de una posición general } \\
\text { y alejada de la realidad. } \\
\text { No se tratan los aspectos }\end{array}$ \\
$\begin{array}{c}\text { Intervención } \\
\text { curriculares relevantes. } \\
\text { (ID) }\end{array}$ & $\begin{array}{l}\text { No se analiza la adecu- } \\
\text { ación de la intervención, } \\
\text { ni las decisiones toma- } \\
\text { das. }\end{array}$ & $\begin{array}{l}\text { Destaca los elementos } \\
\text { relevantes de la acti- } \\
\text { vidad docente (clima, } \\
\text { conducción, ayudas...), } \\
\text { las percepciones y las } \\
\text { emociones vividas, la } \\
\text { actividad del alumna- } \\
\text { do y del currículum. } \\
\text { Identifica los aspectos a a } \\
\text { mejorar. }\end{array}$ & $\begin{array}{l}\text { Destaca los elemtos } \\
\text { relevantes de la actividad } \\
\text { docente (clima, conduc- } \\
\text { ción, ayudas...), las per- } \\
\text { cepciones y las emociones } \\
\text { vividas, la actividad del } \\
\text { alumnado y del currí- } \\
\text { culum. Identifica y hace } \\
\text { propuestas de mejora } \\
\text { concretas para actuacio- } \\
\text { nes futuras. }\end{array}$ \\
\hline
\end{tabular}




\begin{tabular}{|c|l|l|l|l|l|}
\hline $\begin{array}{c}\text { Competencias } \\
\text { Docentes (CD) }\end{array}$ & $\begin{array}{l}\text { No identifica: les compe- } \\
\text { tencias en juego, ni los } \\
\text { recursos (internos/ ex- } \\
\text { ternos) que la situación } \\
\text { requiere movilizar. }\end{array}$ & $\begin{array}{l}\text { Identifica: las compe- } \\
\text { tencias y/o los recursos } \\
\text { movilizados (internos/ } \\
\text { externos). }\end{array}$ & $\begin{array}{l}\text { Identifica y manifiesta } \\
\text { una visión crítica sobre } \\
\text { las competencias y/o los } \\
\text { recursos movilizados (in- } \\
\text { ternos/ externos) y aque- } \\
\text { llos que habría tenido que } \\
\text { movilizar. }\end{array}$ \\
\hline $\begin{array}{c}\text { Oportunidad de } \\
\text { Desarrollo } \\
\text { (OD) }\end{array}$ & $\begin{array}{l}\text { No realizan valoraciones } \\
\text { cobre la propia actua- No hay ninguna } \\
\text { revisión autocrítica o es } \\
\text { débil. }\end{array}$ & $\begin{array}{l}\text { Valora de forma au- } \\
\text { tocrítica la actuación } \\
\text { profesional. Deduce as- } \\
\text { pectos a mejorar como } \\
\text { profesional. }\end{array}$ & $\begin{array}{l}\text { Argumenta, de forma co- } \\
\text { herente sus logros y las } \\
\text { necesidades de cambio } \\
\text { para la mejora profesio- } \\
\text { nal. Plantea objetivos y } \\
\text { vías formativas para el } \\
\text { desarrollo profesional. }\end{array}$ \\
\hline
\end{tabular}

\begin{tabular}{|c|c|c|c|c|c|}
\hline & 1 & 2 & 3 & 4 & 5 \\
\hline \multicolumn{6}{|l|}{ Aspectos formales } \\
\hline $\begin{array}{l}\text { Expresión Escrita } \\
\text { (EE) }\end{array}$ & $\begin{array}{l}\text { Sintaxis poco elaborada, } \\
\text { con errores de construc- } \\
\text { ción que dificultan la } \\
\text { comprensión del dis- } \\
\text { curso. Diversos errores } \\
\text { ortográficos (más de 5). } \\
\text { Lenguaje coloquial y } \\
\text { poco preciso. }\end{array}$ & & $\begin{array}{l}\text { Sintaxis correcta con } \\
\text { algún error ortográfico. } \\
\text { El texto muestra cono- } \\
\text { cimiento terminológico } \\
\text { sobre los marcos teóri- } \\
\text { cos, la docencia y la } \\
\text { normativa. }\end{array}$ & & $\begin{array}{l}\text { La sintaxis y la termi- } \\
\text { nología es elaborada y } \\
\text { pertinente. Sin errores } \\
\text { ortográficos. El texto } \\
\text { muestra conocimiento } \\
\text { terminológico sobre los } \\
\text { marcos teóricos, la do- } \\
\text { cencia y la normativa. } \\
\text { Alta claridad discursiva } \\
\text { al servicio de la temática } \\
\text { y del discurso. }\end{array}$ \\
\hline $\begin{array}{l}\text { Citación y } \\
\text { Bibliografía } \\
\text { (CB) }\end{array}$ & $\begin{array}{l}\text { No hay citación de au- } \\
\text { tores, o no tiene relación } \\
\text { con la temática tratada. } \\
\text { La edición de la bibli- } \\
\text { ografía es incorrecta, o } \\
\text { no hay bibliografía o es } \\
\text { inadecuada. }\end{array}$ & & $\begin{array}{l}\text { La citación y la biblio- } \\
\text { grafía no son correctas } \\
\text { en todos los casos. }\end{array}$ & & $\begin{array}{l}\text { La citación y la biblio- } \\
\text { grafía son correctas y } \\
\text { adecuadas, siguiendo } \\
\text { de forma homogénea la } \\
\text { normativa de citación } \\
(\text { APA, ISO } 690, \ldots) \text {. }\end{array}$ \\
\hline
\end{tabular}

